\title{
Rolandic epilepsy and dyslexia
}

\author{
Epilepsia rolândica e dislexia \\ Ecila P. Oliveira, Marina L. Neri, Livia L. Capelatto, Catarina A. Guimarães, Marilisa M. Guerreiro
}

\begin{abstract}
Objective: Although benign epilepsy with centrotemporal spikes (BECTS) is an idiopathic, age-related epilepsy syndrome with favorable outcome, recent studies have shown impairment in specific neuropsychological tests. The objective of this study was to analyze the comorbidity between dyslexia and BECTS. Method: Thirty-one patients with clinical and electroencephalographic diagnosis of BECTS (group A) and 31 paired children (group B) underwent a language and neuropsychological assessment performed with several standardized protocols. Our findings were categorized as: a) dyslexia; b) other difficulties; c) without difficulties. Our results were compared and statistically analyzed. Results: Our data showed that dyslexia occurred in $19.4 \%$ and other difficulties in $74.2 \%$ of our patients. This was highly significant when compared with the control group $(p<0.001)$. Phonological awareness, writing, reading, arithmetic, and memory tests showed a statistically significant difference when comparing both groups. Conclusion: Our findings show significant evidence of the occurrence of dyslexia in patients with BECTS.
\end{abstract}

Keywords: epilepsy, rolandic, dyslexia, childhood, BECTS.

RESUMO

Objetivo: Apesar da epilepsia benigna da infância com espículas centrotemporais (EBICT) ser uma síndrome epiléptica considerada idiopática, idade-relacionada e de evolução favorável, estudos recentes têm mostrado que essas crianças apresentam prejuízo em testes neuropsicológicos específicos. O objetivo desse estudo foi analisar a comorbidade entre EBICT e dislexia. Método: Trinta e um pacientes com diagnóstico clínico e eletrencefalográfico de EBICT (grupo A) e 31 crianças pareadas (grupo B) foram submetidos à avaliação neuropsicológica e de linguagem com vários protocolos estandardizados. Nossos achados foram categorizados em: a) dislexia; b) outras dificuldades; c) sem dificuldades. Nossos resultados foram comparados e analisados estatisticamente. Resultados: Os dados mostraram que dislexia ocorreu em $19,4 \%$ e outras dificuldades em $74,2 \%$ dos nossos pacientes. Esses números foram altamente significativos quando comparados com o grupo controle $(p<0,001)$. Consciência fonológica, leitura, escrita, aritmética e testes de memória mostraram diferença estatisticamente significante quando foram comparados os dois grupos. Conclusão: Nossos dados mostraram que há evidência da ocorrência de dislexia em pacientes com EBICT.

Palavras-chave: epilepsia, rolândica, dislexia, infância, EBICT.

The comorbidity between epilepsy and language disorder has been a recent issue of interest. Benign childhood epilepsy with centrotemporal spikes (BECTS), also known as rolandic epilepsy, is the most frequent form of epilepsy in childhood and is classified as idiopathic, age-dependent and of benign outcome (spontaneous seizure resolution and absence of brain lesions). Although the prognosis of the seizure is excellent, evidence indicates that BECTS is not so benign, as there may be alterations shown in specific neuropsychological tests, such as language, attention, memory, phonological awareness and school performance.

Deficits in most of these aspects may lead to the diagnosis of certain disorders, including dyslexia. Dyslexia is defined as a reading-related learning disorder of neurobiological origin. It is characterized by difficulty in recognition and fluency in decoding and spelling. This difficulty typically results from a deficit in the phonological component of language that is unexpected in relation to other cognitive abilities considered for the age group ${ }^{1}$.

The prevalence of speech and language disorders in children with epilepsy is still unknown. Moderate forms of language impairment may not be evident during early childhood, arising only at school when the integration of higher cortical functions of language is necessary for the learning process.

Genetics may play a role in the genesis of reading, speech and language disorders and may contribute to the complex

\footnotetext{
Departamento de Neurologia, Faculdade de Ciências Médicas, Universidade Estadual de Campinas, Campinas SP, Brazil.

Correspondence: Marilisa M. Guerreiro; Departamento de Neurologia, Faculdade de Ciências Médicas, Universidade Estadual de Campinas; Rua Tessália Vieira de Camargo, 126; 13083-887 Campinas SP, Brasil; E-mail: mmg@fcm.unicamp.br

Conflict of interest: There is no conflict of interest to declare.

Support: Fundação de Amparo à Pesquisa do Estado de São Paulo (FAPESP 07/59342-7 and 06/56257-6).

Received 10 January 2014; Received in final form 05 July 2014; Accepted 25 July 2014.
} 
etiology of BECTS. Siblings of patients with BECTS are at high risk for reading disorders and speech and language difficulties, thus both patients with BECTS as well as their younger siblings should be evaluated as soon as they enter school ${ }^{2}$.

The aim of this study was to evaluate the presence of dyslexia in patients with BECTS and compare the neuropsychological findings with a control group of children matched for age, gender and educational level.

\section{METHOD}

\section{Participants}

We included 31 consecutive patients (group A) aged between seven and 14 years with BECTS followed at the outpatient childhood epilepsy clinic from March 2008 until April 2010. Children were recruited from a tertiary university hospital, which is a reference center for five million people.

This study was approved by the Ethics Committee of Unicamp Medical School, protocol number 815/2007. Family and/or caregivers have signed a consent form.

The diagnosis of BECTS was reached after clinical examination, EEG and neuroimaging tests (to rule out brain lesions) were performed. Besides the diagnosis, the patients had to have normal neurological, ophthalmologic and audiometric examinations and an IQ at or above 80 .

The following patients were excluded:

1) Those with disturbances in oral and written language resulting from the following, in accordance with the DSM-IV criteria ${ }^{3}$ : pervasive developmental disorders, cerebral palsy, childhood acquired aphasia, mental deficiency or borderline intellectual ability, hearing impairment (including mild conductive hearing loss), and, progressive disorders;

2) Those whose caregivers did not sign the informed consent form.

The control group (group B) consisted of 31 children without neurological impairment, matched for sex, age and socioeconomic level. Children were recruited from regular schools in similar neighborhoods as the patients. Relatives of children with BECTS, children who had IQs below 80, those who were on medication with potential action on the central nervous system, and those with a prior history of neurological problems (such as meningitis, febrile seizures, head trauma with loss of consciousness) and abnormal neurological tests were excluded from the study. Thus, the control group consisted of normal children of the same socio-economic-cultural level as our patients whose parents agreed to participate and signed a consent form.

\section{Instruments \\ Clinical neurological assessment}

Neurological examination was performed by pediatric neurologists and followed our structured protocol.

\section{Neuropsychological assessment}

Neuropsychological assessment was performed by neuropsychologists and included an evaluation of intelligence level and memory and learning processes. The IQ was measured using the full Wechsler Intelligence Scale for Children $3^{\text {rd }}$ edition (WISC-III) ${ }^{4}$.

To evaluate the process of learning and memory we used the Wide Range Assessment of Memory and Learning (WRAML) ${ }^{5}$ which was chosen from compendiums of neuropsychological tests $^{6,7}$ and other studies of epilepsy in children ${ }^{8}$.

The WRAML is an extensive battery used to assess immediate memory, working memory, delayed recall and verbal and visual learning ability. The test covers the age range from five to 17 years and consists of nine subtests: memory for figures, memory for drawings, verbal learning, memory for stories, digital windows, sound-symbol, visual learning, memory for sentences, numbers and letters. The complete application provides indices of verbal memory, visual memory, learning and general memory. For this study we used the scores of verbal memory, visual memory and learning, which were chosen because of a possible occurrence of memory disorder (immediate or working) in patients with dyslexia. In addition, the division between verbal and visual memory could be useful since in phonological dyslexia (which is the diagnostic hypothesis evaluated in this study) it is expected that there is a discrepancy between the two types of memory processes, with a better performance for visual memory.

To facilitate the visualization and statistical analysis instead of using all the usual classifications for memory performance (above average, upper average, average, lower average, below average, etc.) the following classifications were selected: normal and abnormal. It is understood, therefore, that patients with poor performance were those whose memory was rated below average or in lower categories.

\section{Speech evaluation}

\section{Early development history}

An interview with parents or guardians was conducted to learn about history of delay and/or abnormal language development, problems during pregnancy, birth conditions, family history of language delay, language development and hearing behavior; overall cognitive development; development of school learning abilities; motor development; physical development; development of the stomatognathic system and social and environmental conditions.

\section{Language assessment}

Children were evaluated in areas related to phonological awareness, reading and writing, using the following instruments: 
- Academic Performance Test (APT): This test is a psychometric instrument that seeks to offer an objective way of evaluating the fundamental skills for school performance, more specifically, writing, reading and arithmetic. This test was used to assess school performance and compatibility with the chronological age of the children. The APT was designed to evaluate students from first to sixth grades, and is based on criteria developed on the basis of the reality of Brazilian schools ${ }^{9}$.

- Phonological Awareness Test: Metaphonology is related to the abilities to deal intentionally with the phonological properties of speech, for example, to judge whether two speech sounds are similar or not, or say how a given sequence of speech sounds when certain parts are added or removed. Such skills, also known as phonological awareness, are important because international research has demonstrated that children with delays in phonological awareness generally also have delays in reading and writing ${ }^{10}$. According to Chard and Dickson $^{11}$ this ability is critical for proper learning of reading and writing.

- Spontaneous Writing: A sample of the written language of the patients was also obtained through the request to draft a narrative text. Analysis criteria were: spelling and basic rules of accentuation/punctuation, coherence and cohesion in spontaneous text.

It is important to mention that the analysis of the writing sample took into account the educational level of the patient.

\section{Diagnostic hypothesis}

The children studied were divided into three diagnostic categories:

- Dyslexia: characterized by difficulties in phonological awareness, recognition and fluency in decoding and spelling (reading and writing) and poor performance in verbal short-term memory ${ }^{12,13}$.

- Other difficulties: characterized by difficulties (mild to moderate) that may cause impairment in oral language, writing, and consequently in school learning; however, they do not fulfill diagnostic criteria for dyslexia. This category was included so as not to neglect these difficulties.
Table 1. Comparison of dyslexia diagnosis in the group of patients with benign epilepsy with centrotemporal spikes (BECTS) and the control group.

\begin{tabular}{lccc}
\hline \multirow{2}{*}{ Group } & \multicolumn{3}{c}{ DH } \\
\cline { 2 - 4 } & Dyslexia* & OD & WD \\
\hline A & $6(19.4 \%)$ & $23(74.20 \%)$ & $2(6.50 \%)$ \\
B & $0(0.0 \%)$ & $11(35.5 \%)$ & $20(64.50 \%)$ \\
Total & $6(9.70 \%)$ & $34(54.80 \%)$ & $22(35.50 \%)$ \\
\hline \multirow{*}{*}{$p<0.001$. A: Patients with } & BECTS; B: Control group; DH: Diagnostic \\
hypothesis; OD: Other difficulties, WD: Without difficulties. Chi-square test.
\end{tabular}

- Without difficulty: patients who showed no difficulty in any of the tests were placed in this category.

\section{Statistical analysis}

The diagnosis of dyslexia was made by the authors after analyzing the performance of each individual since the ages varied and for each age group a certain classification is expected. After the individual analysis and a conclusive diagnosis, a statistical analysis between groups was performed to establish the statistically significant difference.

The data collected were described and compared using Chi-Square, Mann-Whitney and Fisher Tests to identify possible differences between both groups. Statistical Package for Social Science version 17.0 was used for the statistical analysis of our results with a significance level of 0.05 .

For analysis of the memory data (verbal, visual and learning) 26 patients in group A were compared with 26 children in group B, all matched for sex, age and socioeconomic level. Other five patients did not attend the neuropsychological evaluation.

\section{RESULTS}

Table 1 shows the clinical characteristics of our studied population. The diagnosis of the two groups (A and $\mathrm{B}$ ) is laid out in Table 1, which shows a statistically significant difference $(p<0.001)$ for the presence of dyslexia in six of the 31 patients in group A and in none of the children in the control group. Table 2 shows in detail the significant tests utilized for dyslexia diagnosis in those six patients. Other difficulties were found in 23 patients in group A but only in 11 children in group B $(\mathrm{p}<0.001)$.

Table 2. Significant tests for dyslexia diagnosis.

\begin{tabular}{|c|c|c|c|c|c|c|c|}
\hline & PAT (total-40) & Writing & Reading & Arithmetic & Verbal memory & Visual memory & Learning memory \\
\hline A1 & 12 & inferior & inferior & medium & abnormal & normal & normal \\
\hline A2 & 17 & inferior & inferior & medium & abnormal & normal & abnormal \\
\hline A3 & 29 & inferior & inferior & superior & abnormal & normal & abnormal \\
\hline A4 & 32 & inferior & medium & medium & abnormal & normal & normal \\
\hline A5 & 15 & inferior & inferior & medium & abnormal & normal & abnormal \\
\hline A6 & 33 & inferior & medium & superior & abnormal & normal & normal \\
\hline
\end{tabular}

A: Patients with benign epilepsy with centrotemporal spikes (BECTS); PAT: Phonological awareness test. 
Table 3. Comparison of the phonological awareness test in the group of patients with BECTS and the control group.

\begin{tabular}{cccccc}
\hline & Groups & N & Mean & Standard-deviation & $p^{*}$ \\
\hline PAT & A & 31 & 73.70 & 20.60 & 0.001 \\
& B & 31 & 86.93 & 4.48 & \\
\hline
\end{tabular}

*Mann-Whitney test. A: Patients with benign epilepsy with centrotemporal spikes (BECTS); B: Control group; PAT: Phonological awareness test.

The values in the Phonological Awareness Test (Table 3) also showed a statistically significant difference when comparing data from group A and group B $(\mathrm{p}<0.001)$.

To assess school performance and compare it to children of similar age and education, three subtests were applied: writing, reading, and arithmetic. Subtests in reading and writing showed a significant difference (Table 4) between the group of children with epilepsy and the control group $(\mathrm{p}<0.001$ and $\mathrm{p}<0.001$, respectively), thereby strengthening reading and writing disorders as the most expected comorbidities of BECTS. There was evidence of lower performance in reading and writing in all patients with dyslexia and better performance in arithmetic ( $p=0.187$ ), but without statistical significance.

Analyzing the memory data (Table 5), a statistically significant difference was found only with respect to verbal memory $(\mathrm{p}<0.037)$ in group $\mathrm{A}$, while for visual memory ( $\mathrm{p}=0.554)$ and learning $(\mathrm{p}=0.097)$ the correlation was not found to be statistically significant.

\section{DISCUSSION}

Our findings show significant evidence of the occurrence of dyslexia in patients with BECTS. Of the 31 patients, six (19.4\%) had a diagnosis of dyslexia. The main finding of our study contributes significantly to the understanding of school performance of children with BECTS, the relevance of which should be a priority for the pediatric neurologist and other healthcare staff who work with these children. The great contribution, however, lies not only in finding abnormalities in patients with BECTS, but in some important details that will be discussed.

Many studies have addressed the relationship between oral language (especially in relation to praxis) and written language $\mathrm{e}^{14,15,16,17,18}$. However, these studies did not use the term dyslexia for the abnormalities found in children with BECTS. In the study by Vinayan et al. ${ }^{19}, 50$ children with BECTS were evaluated and $27(54 \%)$ were found to have "educational problems," a term defined by the authors as difficulty in reading, spelling and auditory discrimination. Papavasiliou et al. ${ }^{20}$ evaluated 32 patients with BECTS in order to investigate their written language skills. They found that $48 \%$ of patients had signs indicating dyslexia. Of these 14 patients, three were considered dyslexic.

Thus, our finding of $19.4 \%$ was highly significant, not only because of the value, but due to the fact that all the required tests to fulfill the diagnosis of dyslexia were performed. In this study, an extensive and comprehensive methodology was applied through specific and detailed evaluations conducted by a multidisciplinary team.

It should again be emphasized that, when viewed alone, the results of the tests do not allow the precise diagnosis of

Table 4. Comparison of writing, reading and arithmetic in the group of patients with benign epilepsy with centrotemporal spikes (BECTS) and the control group.

\begin{tabular}{|c|c|c|c|c|c|}
\hline APT & Group & Inferior & Medium & Superior & $p^{*}$ \\
\hline \multirow[t]{2}{*}{ Writing } & A & $20(64.5 \%)$ & $8(25.8 \%)$ & $3(9.7 \%)$ & 0.001 \\
\hline & $\mathrm{B}$ & $0(0.0 \%)$ & $12(38.7 \%)$ & 19 (61.3\%) & \\
\hline \multirow[t]{2}{*}{ Arithmetic } & $A$ & $9(29.0 \%)$ & $19(61.3 \%)$ & $3(9.7 \%)$ & 0.187 \\
\hline & $B$ & 7 (22.6\%) & $15(48.4 \%)$ & $9(29.0 \%)$ & \\
\hline \multirow[t]{2}{*}{ Reading } & A & $18(58.1 \%)$ & $5(16.1 \%)$ & $8(25.8 \%)$ & 0.001 \\
\hline & $B$ & $0(0.0 \%)$ & $12(38.7 \%)$ & $19(61.3 \%)$ & \\
\hline
\end{tabular}

*Mann-Whitney test. A: Patients with BECTS; B: Control group; APT: Academic performance test.

Table 5. Comparison of memory in the group of patients with benign epilepsy with centrotemporal spikes (BECTS) and the control group.

\begin{tabular}{|c|c|c|c|c|}
\hline Memory & Group & Normal & Abnormal & $p^{*}$ \\
\hline \multirow[t]{2}{*}{ Verbal } & $A$ & $20(76.9 \%)$ & $6(23.1 \%)$ & 0.037 \\
\hline & B & $25(96.1 \%)$ & $1(3.9 \%)$ & \\
\hline \multirow[t]{2}{*}{ Visual } & $A$ & 22 (84.0\%) & $4(16.0 \%)$ & 0.554 \\
\hline & B & $25(96.1 \%)$ & $1(3.9 \%)$ & \\
\hline Learning & $\begin{array}{l}A \\
B\end{array}$ & $\begin{array}{l}18(69.2 \%) \\
20(76.9 \%)\end{array}$ & $\begin{array}{l}8(30.8 \%) \\
6(23.1 \%)\end{array}$ & 0.097 \\
\hline
\end{tabular}

*Fisher test. A: patients with BECTS; B: Control group. 
dyslexia. Miziara ${ }^{21}$ found a statistically significant difference between a group of patients with BECTS and their control group with regard to the results of the APT, one of the tests used in our study. Approximately $65 \%$ of patients with BECTS showed lower performance in the APT.

Another factor that was addressed very conscientiously in this study was the selection of the control group. The selection of a suitable control group requires careful consideration. The use of siblings without BECTS acting as control subjects has been rejected due to the high incidence of EEG abnormalities in first-degree relatives. The use of a "best friend" was also not possible because the patients' families tended to be resistant to disclosing the epilepsy diagnosis to friends. Recruitment from regular schools avoided the above difficulties and thus was used for this study. Group B contained the same number of participants as group A and the subjects were all matched for sex, age and socioeconomic level. This is not always the case. Clarke et al. ${ }^{2}$ studied 29 patients with BECTS and compared them with a control group of 24 children, but without achieving the same degree of matching as this study. The authors of that study found that $55 \%$ of patients and $16 \%$ of controls had a reading disorder.

There is no denying the contribution of previous studies, but we consider that simply identifying the presence or absence of a disorder or difficulty is not enough to provide sufficient information for the purpose of efficient intervention and appropriate educational planning.

The tests used in our study for the diagnosis of dyslexia will now be discussed.

Phonological awareness is essential in the identification of dyslexia. The scores shown in Table 2 varied according to the age of the patient evaluated. For this reason, each case had to be analyzed individually. Our data support previous studies, such as that by Northcott et al. ${ }^{22}$ in which children with BECTS also scored significantly lower than the normative data on four of the five subtests of phonological awareness.

Comparing the performance of dyslexic children and children with BECTS in skills related to learning, the impediment is most often found in areas of reading, writing and spelling ${ }^{20,23}$. The children with low scores in phonological awareness have difficulty reading and spelling, which underscores the important relationship between phonological awareness and reading skills. This is one of the reasons why reading and writing skills are also important to confirm or exclude the diagnosis of dyslexia.

Patients diagnosed with dyslexia had a lower performance in reading and writing and an average performance in arithmetic, a condition consistent with the diagnosis of dyslexia. An average or above average score in arithmetic is, in many cases, what differentiates dyslexia from other learning disorders.

Finally, to confirm or exclude the diagnosis of dyslexia in our patients, neuropsychological tests involving memory types were considered. In our six patients diagnosed with dyslexia, the worst scores found were related to verbal memory and learning, while visual memory remained within average or above average (findings similar to the control group), in agreement with other studies ${ }^{16,24}$. It is important to emphasize that the memory data alone is not sufficient for this diagnosis. It is necessary to correlate the results of language assessment and neuropsychological assessment.

Thus, we conclude that children with BECTS may not only present with disturbances in oral language, writing and memory, but can also have disorders such as dyslexia and this diagnosis is often overlooked.

Rolandic epilepsy patients therefore may benefit from professional assessment by psychologists and speech therapists at diagnosis and language evaluation should always be investigated, especially in school-age children, regardless of seizure control.

\section{References}

1. Lyon GR, Shaywitz SE, Shaywitz BA. A definition of dyslexia. Ann Dyslexia. 2003;53(1):1-14. http://dx.doi.org/10.1007/s11881-003-0001-9

2. Clarke T, Strug LJ, Murphy PL, Bali B, Carvalho J, Foster S et al. High risk of reading disability and speech sound disorder in rolandic epilepsy families: case-control study. Epilepsia. 2007;48(12):2258-65. http://dx.doi.org/10.1111/j.1528-1167.2007.01276.x

3. American Psychiatric Association. Diagnostic and statistical manual of mental disorders (DSM-IV). 4th ed. Washington: American Psychiatric Association; 1994.

4. Wechsler D. WISCIII: Wechsler Intelligence Scale for Children: manual. 3th ed. 1991. São Paulo: Casa do Psicólogo; 2002.

5. Sheslow D, Adams W. Wide range assessment of memory and learning: administration manual. Willmington: Wide Range; 1990.

6. Lezak MD. Neuropsychology assessment. New York: Oxford University Press; 1995

7. Spreen O, Strauss E. Compedium of neuropsychological tests. New York: Oxford University Press; 1991.
8. Guimarães CA, Li LM, Rzezak P, Fuentes D, Franzon RC, Montenegro MA et al. Temporal lobe epilepsy in childhood: comprehensive neuropsychological assessment. J Child Neurol. 2007;22(7):836-40. http://dx.doi.org/10.1177/0883073807304701

9. Stein LM. TDE - Teste de desempenho escolar. São Paulo: Casa do Psicólogo; 1994.

10. Capovilla AGS, Capovilla FC, editores. Problemas de leitura e escrita. São Paulo: Memnon; 2003. Prova de consciência fonológica; p. 233-7.

11. Chard DJ, Dickson SV. Phonological awareness: instructional and assessment guidelines. Interv Sch Clin. 1999;34(5):261-70.

12. Frederickson N Reason R. Phonological assessment of specific learning difficulties. Ed Child Psychol 1995;12:6-17.

13. Oliveira EPM, Hage SRV, Guimarães CA, Brandão-Almeida I, LopesCendes I, Guerreiro CA et al. Characterization of language and reading skills in familial polymicrogyria. Brain Dev. 2008;30(4):254-60. http://dx.doi.org/10.1016/j.braindev.2007.08.010 
14. Brown SW, Reynolds EH. Cognitive impairment in epileptic patients. In: Reynolds EH, Trimble MR, editors. Epilepsy and psychiatry. Edinburgh: Churchill Livingstone; 1981. p. 34-48.

15. D’Alessandro P, Piccirilli M, Tiacci C, Ibba A, Maiotti M, Sciarma T et al. Neuropsychological features of benign partial epilepsy in children. Ital J Neurol Sci. 1990;11(3):265-9. http://dx.doi.org/10.1007/bf02333856

16. Croona $\mathrm{C}$, Kihlgren $\mathrm{M}$, Lundberg $\mathrm{S}$, Eeg-Olofsson KE. Neuropsychological findings in children with benign childhood epilepsy with centrotemporal spikes. Dev Med Child Neurol. 1999;41(12):813-8. http://dx.doi.org/10.1017/s0012162299001620

17. Fonseca LC, Tedrus GMAS, Tonelotto JMF, Antunes TA, Chiodi MG. Desempenho escolar em crianças com epilepsia benigna da infância com pontas centrotemporais. Arq Neuropsiquiatr. 2004;62(2B):459-62. http://dx.doi.org/10.1590/s0004-282x2004000300015

18. Lindgren A, Kihlgren M, Melin L, Croona C, Lundberg S, Eeg-Olofsson O. Development of cognitive function in children with RE. Epilepsy Behav. 2004;5(6):903-10. http://dx.doi.org/10.1016/j.yebeh.2004.08.004

19. Vinayan KP, Biji V, Thomas SV. Educational problems with underlying neuropsychological impairment are common in children with benign epilepsy of childhood with centrotemporal spikes (BECTS). Seizure. 2005;14(3):207-12. http://dx.doi.org/10.1016/j.seizure.2005.01.009

20. Papavisiliou A, Mattheou H, Bazigou H, Kotsalis C, Paraskevoulakos E. Written language skills in children with benign childhood epilepsy with centrotemporal spikes. Epilepsy Behav. 2005;6(1):50-8. http://dx.doi.org/10.1016/j.yebeh.2004.09.008

21. Miziara CSMG. Avaliação das funções cognitivas na epilepsia focal benigna da infância com descargas centrotemporais [thesis]. São Paulo: Universidade de São Paulo; 2003.

22. Northcott E, Connolly AM, Berroya A, Sabaz M, Mclntyre J, Christie J et al. The neuropsychological and language profile of children with benign rolandic epilepsy. Epilepsia. 2005;46(6):924-30. http://dx.doi.org/10.1111/j.1528-1167.2005.62304.x

23. Staden U, Isaaca E, Boyd SG, Brandl U, Neville BGR. Language dysfunction in children with rolandic epilepsy. Neuropediatrics. 1998;29(5):242-8. http://dx.doi.org/10.1055/s-2007-973569

24. Metz-Lutz MN, Kleitz C, Saint Martin A, Massa R, Hirsch E, Marescaux C. Cognitive development in benign focal epilepsies of childhood. Dev Neurosci. 1999;21(3-5):182-90. http://dx.doi.org/10.1159/000017397 